\title{
Agir concrètement et en parler
}

\section{Remo Osterwalder}

Dr med., membre du Comité central de la FMH, responsable du département Médecins en libre-pratique

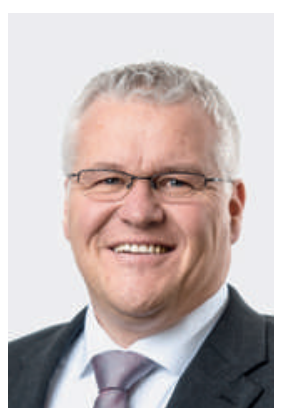

Tout vient à point à qui sait attendre: en juin dernier, l’unité opérationnelle du département Médecins en libre-pratique a vu le jour au sein du Secrétariat général de la FMH. Il est primordial à l'heure actuelle d'analyser la situation des médecins installés, notamment en ce qui concerne les reprises de cabinets mais aussi les modèles de prise en charge qui doivent être adaptés aux attentes des médecins de demain. Les nouvelles ressources à disposition ont permis de relancer le groupe de travail «soins de base ambulatoires», initialement mandaté par l'Assemblée des délégués. Ce groupe de travail doit permettre, entre autres, de passer en revue le point de vue et les attentes des médecins installés afin d'y répondre par des projets ciblés. Actuellement, il se penche sur des thèmes tels que le manque de clarté dans l'évolution actuelle des cabinets, la génération $Y$ et les modèles innovants de prise en charge.

\section{Quel est l'impact du manque de clarté dans l'évolution des cabinets?}

Nous voulons continuer dans le futur de nous investir en faveur de l'indépendance thérapeutique et économique des médecins installés car, d'une part, l'influence accrue d'institutions non médicales est devenue une tendance fortement perceptible dans le secteur de la

\section{Sans savoir où nous sommes aujourd'hui,} comment prédire où nous irons demain?

\footnotetext{
1 Schmidt CE, Möller J, Schmidt K, Gerbershagen MU, Wappler F, Limmroth V, et al. Generation Y: Rekrutierung, Entwicklung und Bindung. Anaesthesist. 2011; 60(6):517-24. doi: 10.1007/ s00101-011-1886-z
}

santé et, d'autre part, le concept classique du cabinet médical est sujet à de nombreuses évolutions. Après le départ à la retraite de leur propriétaire, les cabinets médicaux sont de plus en plus souvent rachetés par des franchises ou des réseaux de soins. Pour les patients, il est cependant primordial que ces nouvelles entités soient dépourvues de tout conflit d'intérêts. Mais comment le savoir? Sans une standardisation de la transparence, le patient n'a pratiquement aucune possibilité de s'en assurer.

\section{Génération Y - une notion qui a le vent en poupe}

Aujourd'hui, pour rester dans la course, il faut se confronter aux attentes de la génération Y qui n'invoque qu'un seul mot d'ordre: flexibilité. En termes de concurrence, l'avantage reviendra à celui qui aura fait

\section{Façonner activement son avenir peut aussi} signifier se tromper.

une bonne analyse de la situation car le recrutement et le maintien de collaborateurs Y constituent un facteur décisif du succès économique d'un hôpital ou d'un cabinet médical [1]. Dès lors que la connaissance de leurs attentes et des mesures à prendre revêt une importance considérable, une pléthore d'études se penchent sur cette génération Y et constituent par ailleurs, pour les plus anciens d'entre nous, des sources intarissables à lire pour en apprendre davantage sur la relève. Mais quelle est la valeur et la fiabilité de ces études ? Par ailleurs, elles traitent le plus souvent de la génération $\mathrm{Y}$ en général, alors que la FMH s'intéresse aux caractéristiques des médecins en particulier. En effet, ce n'est qu'une connaissance précise des besoins de ce groupe spécifique qui permettra d'apporter des réponses adéquates. C'est pourquoi nous investissons une part importante de notre travail dans le projet génération Y.

\section{Modèles de prise en charge - lesquels ont du succès?}

Notre division plaide pour le soutien, l'encouragement et le développement de modèles innovants. Dans un premier temps, il s'agit donc d'établir une vue d'ensemble de ce qui existe déjà et fonctionne avec succès. Il n'y a pas qu'un seul et unique modèle, bien au contraire. L'individualité des modèles régionaux répond, en matière de besoins et d'exigences, à la diversité et à la spécificité de nos patients. Les structures existantes comme les plateformes d'informations devraient être encouragées. 